$16^{\text {th }}$ International Congress of Metrology, 06002 (2013)

DOI: $10.1051 /$ metrology/201306002

(c) Owned by the authors, published by EDP Sciences, 2013

\title{
Modélisation et évaluation de l'incertitude de mesure lors de l'utilisation de MMT avec des pièces déformables
}

\author{
Stéphane Raynaud ${ }^{1}$, Valéry Wolff ${ }^{2, a}$ and Tin Tran Dinh ${ }^{3}$ \\ ${ }^{1}$ MIP2, Département GMC, Laboratoire MIP2 - Métrologie Qualité, INSA de Lyon, France \\ ${ }^{2}$ IUT LYON1, Département GMP, Université Lyon 1, France \\ ${ }^{3}$ LAMCOS, INSA de Lyon, France
}

\begin{abstract}
Résumé. Lors de l'étape du contrôle des pièces, de nombreux paramètres influencent le résultat de la mesure. Les pièces sont habituellement considérées comme des corps rigides quasi-indéformables. Les paramètres influents les plus couramment étudiés concernent le palpeur et la machine. Ces études prennent en compte les problèmes d'étalonnage et de calibration des palpeurs d'une part, et les calculs de dilatation d'autre part. Dans le cas des pièces déformables, ces hypothèses de travail ne sont pas satisfaisantes. La déformation des pièces lors du bridage sur le montage de contrôle n'est alors pas négligeable au regard des résultats de mesure attendus. Dans cet article, nous proposons d'étudier les effets de la déformation d'une pièce souple sur les résultats de la mesure. La première étape de cette étude consiste à modéliser le comportement de la pièce flexible lors de sa mise en place et de son bridage sur le montage de contrôle de métrologie. Les défauts géométriques du montage de contrôle sont représentés par l'utilisation de tolérances ISO dans le logiciel 3DCS et la déformation de la pièce est calculée en utilisant les méthodes par élément finis. Le cas d'application est une simple tôle pliée. Cette étude doit confirmer la concordance entre le modèle numérique et une maquette réelle réalisée et mesurée sur MMT.
\end{abstract}

\section{Introduction}

La réalisation de pièces industrielles conformes aux spécifications ne peut être envisagée que si le processus de contrôle est maîtrisé. Lors de cette étape de mesure et de validation, de nombreux paramètres influencent le résultat.

L'étude présentée ici concerne le cas particulier des pièces déformables [1][2][3][4]. Il s'agit de prendre en compte les problèmes liés à la déformation de la pièce lors du processus de métrologie.

L'objectif de cette étude est de comparer les résultats d'une simulation numérique prenant en compte les déformations de la pièce lors de sa mesure avec une maquette réalisée et mesurée.

\section{Problématique}

Les pièces sont habituellement considérées comme des corps rigides quasi-indéformables. Cette hypothèse n'est évidement pas adaptée à l'ensemble des pièces mesurées dans les différents secteurs d'activité. On trouve deux grandes catégories de pièces déformables dans l'industrie mécanique : les pièces de tôlerie (métaux en feuilles) et les pièces plastiques (pièces minces injectées). Ces pièces se retrouvent tout particulièrement dans l'industrie automobile. Les pièces concernées vont de la carrosserie extérieure du véhicule (tôlerie), jusqu'aux éléments d'habillage intérieurs comme le tableau de bord (injection plastique). Un exemple de pièce déformable représentatif de la problématique des industriels est le bouclier avant des véhicules. Cette pièce très souple se déforme très facilement sous son propre poids : pièce déformable à l'état libre. Elle se déforme également lors du montage sur le véhicule pour se conformer aux différents points d'ancrage prévus. Dans ce dernier cas, il s'agit de déformation de la géométrie sous contraintes.

Lorsqu'une pièce se déforme sans contrainte autre que son propre poids, elle n'est pas mesurable de manière fiable à l'état libre. Il faut définir et réaliser des montages de contrôle de pièces spécifiques. Ces montages doivent permettre de conformer la pièce afin qu'elle prenne la forme (déformée sous contraintes) qu'elle aura lors de son utilisation finale. Pour cela, on s'appuie sur des points de positionnement et des points de bridage. Les montages de contrôle sont le plus souvent hyperstatiques. L'étude et la réalisation de ce type de montage de contrôle est d'un coût non négligeable pour l'entreprise.

Dans le domaine des pièces automobile, qu'elles soient de tôlerie ou plastiques, il serait intéressant de pouvoir remplacer ces montages de contrôle de pièces déformables par une simulation numérique. L'économie concernant

\footnotetext{
${ }^{\mathrm{a}}$ Corresponding author: valery.wolff@univ-lyon1.fr
} 
l'équipement s'accompagnerait sans aucun doute également d'un gain de temps important sur le processus de mise au point et de validation des pièces fabriquées. Cette étude est une première étape en ce sens.

\subsection{Contexte}

On trouve un grand nombre de travaux consacrés aux défauts observés lors du processus de mesure. Ces recherches prennent en compte les défauts dus aux moyens de mesures utilisés : il s'agit souvent de caractériser les machines ou les capteurs. Les méthodes utilisées sont basées sur des calculs de dispersions, de justesse et de fidélité des instruments de mesure. Les calculs peuvent être analytiques ou statistiques.

Dans le premier cas (méthodes analytiques), il s'agit de s'appuyer sur les lois physiques de comportement des différents éléments de la chaine de mesure. Par exemple, de nombreuses études prennent en compte les déformations sous l'influence des variations de température : phénomènes de dilatation des pièces ou des éléments de la machine.

Dans le second cas, on utilise par exemple la valeur de dispersion statistique basée sur des valeurs expérimentales (retour d'expérience). Pour une loi normale de probabilité, on applique la méthode six-sigma où la dispersion correspond à 99,73\% des valeurs pour une étendue égale à six fois l'écart-type. On détermine alors l'indicateur de capabilité $\mathrm{C}_{\mathrm{mc}}$ : capabilité du moyen de mesure.

$$
C_{m c}=\frac{I T}{6 \times \sigma_{\text {instrument }}}
$$

Dans le cadre de cette étude, nous utilisons les méthodes basées sur dispersions statistiques.

\subsection{Outil logiciel : 3DCS}

Il s'agit d'un logiciel associé au système de CAO CATIA. Habituellement, il est utilisé pour étudier la fonctionnalité d'un mécanisme. Il permet d'évaluer dès la conception les effets du tolérancement dimensionnel et géométrique sur les jeux fonctionnels et contacts souhaités pour le mécanisme [5]. Il intègre également des fonctionnalités pour prendre en compte le comportement des pièces non rigides en utilisant le module complémentaire de calculs par éléments finis.

\subsubsection{Le logiciel 3DCS}

Intégré à CATIA, il utilise la géométrie des pièces issues de la CAO ainsi que le tolérancement 3D associé (les annotations FT\&A*, Figure 1). L'assemblage doit être modélisé en utilisant des mises en position hiérarchisées des solides appelées «MOVES» (Figure 2). Elles se distinguent des liaisons mécaniques classiques (liaison pivot, rotule, ...) que l'on trouve habituellement dans les modules assemblages des logiciels de CAO. Dans 3DCS, chaque MOVE est indépendant de ceux qui le précèdent. Cette démarche, surprenante au premier abord, est au final

* FT\&A : Functional Tolerances \& Annotation bien adapté à la modélisation de méthodologie pratique et surtout à la prise en compte des processus d'assemblage ou de fabrication. Dans notre cas, nous l'utilisons pour simuler le processus de mesure.

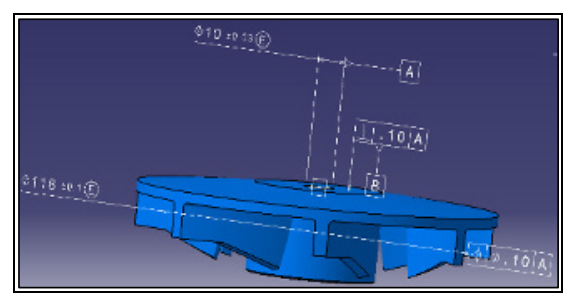

Figure 1 : module FT\&A de Catia

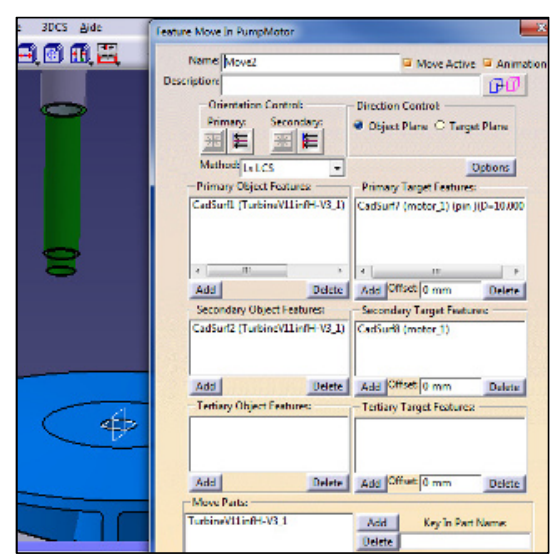

Figure 2 : Les fonctions MOVE de 3DCS

\subsubsection{Méthode de simulation}

La méthode utilisée est basée sur la simulation de MonteCarlo. Le logiciel génère un nombre élevé d'assemblages différents à partir de pièces pour lesquelles chaque spécification dimensionnelle ou géométrique est générée aléatoirement en suivant des lois de distribution (Figure 3) statistique conformes au processus de fabrication [5] (loi normale , loi uniforme, loi de Rayleigh ...) envisagé et au tolérancement choisi par le concepteur (annotations FTA).

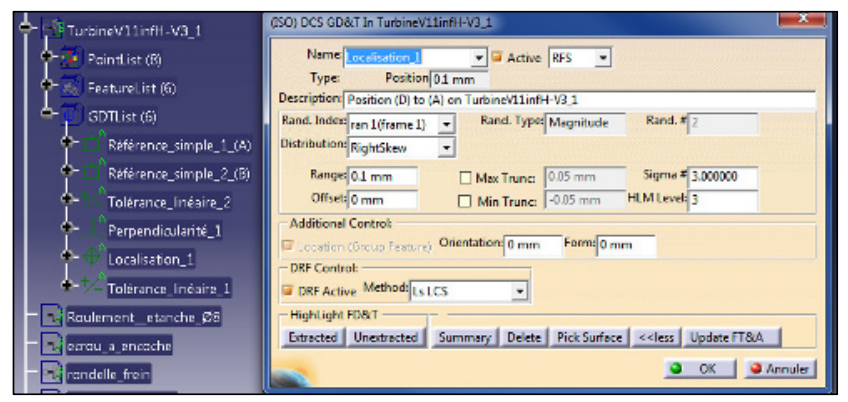

Figure 3 : Loi de distributions dans 3DCS

Dans le cas particulier de notre utilisation pour effectuer des mesures virtuelles, il est important de noter que 3DCS génère pour chaque point des positions aléatoires.

Par exemple, un défaut de planéité appliqué sous forme d'annotation FTA à une surface entraîne un maillage de la surface. Chaque point est ensuite affecté des déplacements 
possibles conformes à la définition de la spécification. Dans le cas de la planéité, un point du maillage peut varier de position en suivant la normale au plan et en respectant une distribution aléatoire normale ayant une étendue à plus ou moins un demi-défaut de planéité.

\subsection{Hypothèse de travail}

Les pièces déformables comme le bouclier cité précédemment doivent être calculées en simulation numérique en utilisant des méthodes de calcul par éléments finis appliquant des calculs non linéaires (hyperélasticité). Le comportement de ces matériaux complexes (parfois chargés) est en effet hors du domaine élastique linéaire classique.

Les travaux présentés ici sont pour l'instant limités à un comportement élastique basé sur le module d'Young et le coefficient de Poisson. Cette hypothèse de travail est principalement imposée par les limitations actuelles de l'un des logiciels utilisé pour la mise en œuvre (3DCS), qui ne prend pas en compte les paramètres de MooneyRivlin concernant l'hyper-élasticité incompressible. Le choix de la "pièce test » a été fait pour pouvoir appliquer les hypothèses de travail suivantes :

- La déformation globale de la pièce sera considérée comme un grand déplacement (extrémité de la pièce sur un appui réglable)

- La déformation locale de la pièce sera considérée comme linéaire élastique

\section{Etude}

Industriellement, les pièces sont produites avec des défauts géométriques et des défauts dimensionnels. Le montage de contrôle permet pour chacune de ces pièces (ou éventuellement pour un échantillon en cas de contrôle statistique), de valider le respect du tolérancement demandé par le bureau d'études. On a donc plusieurs pièces à contrôler sur un unique montage de validation.

Pour notre étude, il était impossible de disposer d'un lot de pièces représentatif d'une production de série associé à un montage de contrôle. Il n'était pas non plus envisageable de fabriquer suffisamment de pièces test en maitrisant les dispersions de fabrication et à un coût raisonnable. L'étude se fera donc avec une pièce test unique et un montage de contrôle réglable. Le procédé est donc inversé : au lieu de calculer la déformée de nombreuses pièces de dimensions variables pour les conformer à un montage de dimension fixe, nous calculons la déformée d'une pièce unique qui s'adapte à la géométrie d'un montage de dimension variable. Les résultats obtenus sont similaires et transférables à la démarche inverse.

\subsection{Définition de la pièce test}

La pièce choisie pour cette étude est une simple tôle ajourée pliée (Figure 4). Elle a été choisie métallique afin de pouvoir appliquer les hypothèses d'élasticité linéaire sur le déplacement appliqué lors de l'essai. Les découpes sont asymétriques afin de provoquer une déformation de type torsion en plus de la déformation de type flexion.

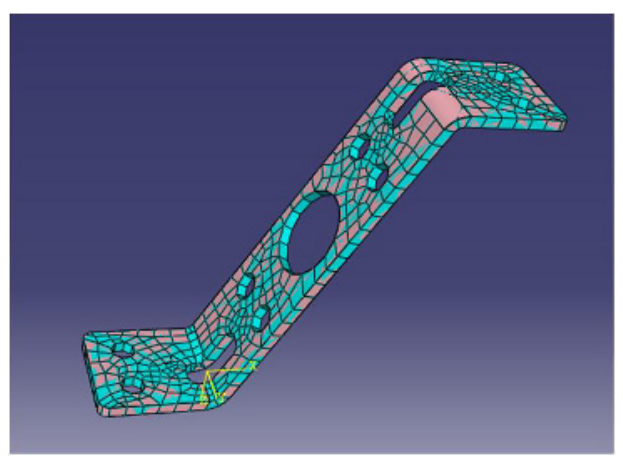

Figure 4. La pièce test utilisée

\subsection{Méthodologie pratique}

La démarche utilisée se compose de 3 étapes :

- La modélisation de la pièce et du montage de contrôle, la génération de la matrice de raideur pour les calculs de déformation par FEM. [2][3][6][7]

- Les mesures virtuelles sur le modèle numérique déformé. - Mesure de la maquette sur MMT et comparaison des résultats.

\subsubsection{Modélisation}

La première étape de notre travail consiste à utiliser un logiciel de calcul par éléments finis afin d'obtenir les données d'entrée pour la simulation numérique de la mesure. Le logiciel Abaqus (Figure 5) nous fourni les matrices de raideur et le maillage [4][6][8][9] qui seront utilisés ensuite par le module FEA Compliant Modeler du logiciel 3DCS (utilisé pour la simulation du tolérancement et de ses effets sur des mesures).

Le calcul par éléments finis permet également de déterminer la force à appliquer pour obtenir le déplacement correspondant à l'expérimentation $[1][2][3][10][11]$. Dans le cas de cette pièce test, le déplacement (sur la maquette physique) a été réglé sur 4 valeurs : $0,1,2$ et $3 \mathrm{~mm}$ de déplacement du point d'appui [4].

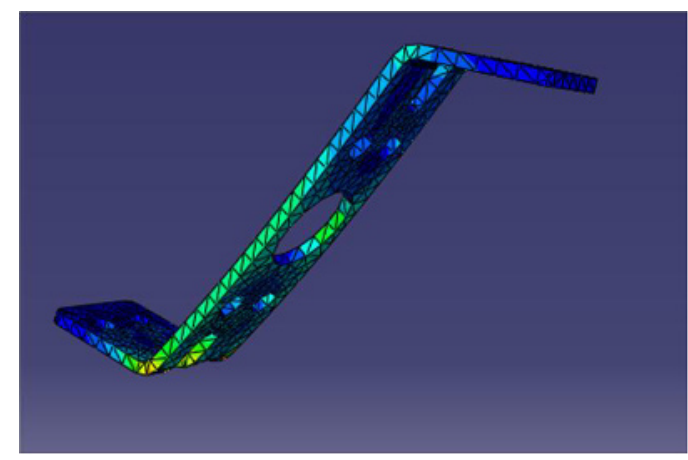

Figure 5. Maillage et calcul FEM sur la pièce test

\subsubsection{Mesures virtuelles}


L'utilisation du module FEA Compliant Modeler de 3DCS [5] permet d'obtenir la géométrie après déformation en appliquant une force ou un déplacement sur un point particulier. Sur la pièce test, nous avons définis 4 mesures à étudier.

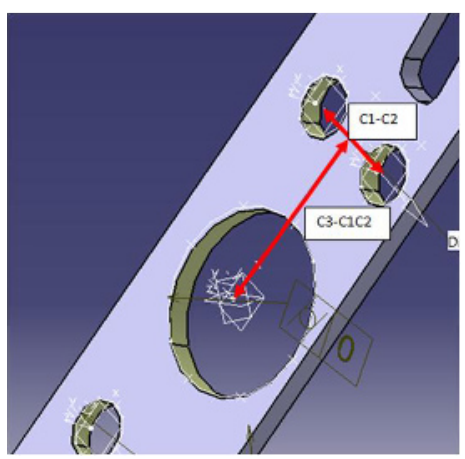

Figure 6. Les mesures sur 3DCS

La pièce présente 5 alésages sur la face inclinée (Figure 5). Nous avons choisi de mesurer des distances concernant 3 d'entre eux. Une mesure «horizontale» entre les 2 petits alésages du haut de la pièce sera notée C1-C2 (Figure 6). Pour obtenir une distance correspondant à la définition d'une localisation en cotation ISO, la distance notée $\mathrm{C} 3-\mathrm{C} 1 \mathrm{C} 2$ est obtenue après construction d'une droite passant par $\mathrm{C} 1$ et $\mathrm{C} 2$, et mesure de la distance entre le centre du cercle $\mathrm{C} 3$ et la droite précédente.

Lorsque la mesure de la distance n'est pas possible en utilisant directement des fonctions intégrées au logiciel 3DCS, nous avons utilisé Metrolog XG pour effectuer les calculs. Les données d'entrée pour les calculs sont des nuages de points exportés depuis 3DCS et importés [5]. Ces points issus du modèle CAO sont des «feature points ». Cela signifie qu'ils sont associés à une surface et qu'ils subissent les déplacements liés au tolérancement et à la déformation de la pièce.

Par exemple, dans le cas de la mesure du défaut de planéité de la surface inclinée, le logiciel 3DCS permet d'obtenir les positions des points choisis sur la surface en obtenant leurs coordonnées dans un référentiel XYZ choisi après application de la déformation de la pièce et des dispersions associées au tolérancement. Ces points sont ensuite importés dans le logiciel de métrologie Metrolog XG et les calculs sont effectués en appliquant le critère des moindres carrés au nuage de points pour déterminer le plan associé et obtenir le défaut de forme.

\subsubsection{Mesure de la maquette}

Les mesures réelles de la pièce se font sur une machine à mesurer tridimensionnelle équipée du logiciel Metrolog XG (Figure 7). L’opération réalisée lors des mesures virtuelles présentées au paragraphe précédent et consistant à effectuer les calculs à l'aide du logiciel Metrolog XG a permis de faire en sorte que les méthodes soient identiques pour le modèle numérique et pour la pièce réelle mesurée sur MMT [4].

Pour chaque valeur de déplacement choisie pour l'expérimentation, le déplacement de l'appui a été validé par une mesure. Ensuite, la pièce a été mesurée 10 fois dans chaque configuration afin d'éliminer le défaut de fidélité de la machine. Les résultats présentés sur les graphes dans le paragraphe suivant sont les moyennes obtenues lors de cette répétition de mesures.

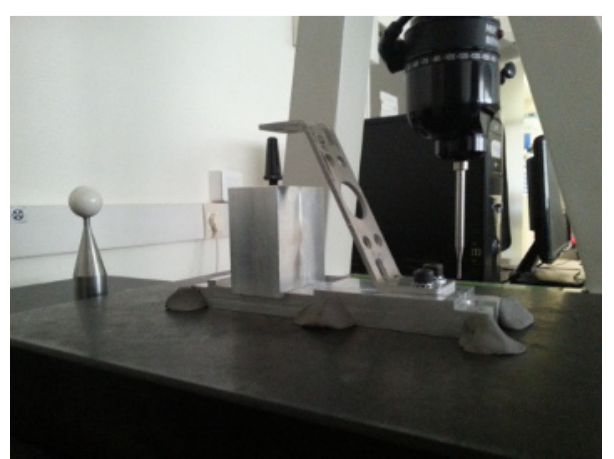

Figure 7. Montage de mesure

\section{Résultats}

Les valeurs obtenues lors de la mesure de la pièce réelle sur son montage sont appelées par la suite: mesures expérimentales. Les résultats des mesures virtuelles regroupent des valeurs obtenues directement depuis le logiciel 3DCS (ex : distance entre 2 centres de cercles) et des valeurs obtenues en appliquant une méthode de calcul sur le logiciel Metrolog XG (ex : mesure de l'angle entre le plan associé par les moindres carrés et le plan XY du repère principal). Ces valeurs seront dénommées : mesures virtuelles. Les résultats de la comparaison entre les mesures expérimentales et les mesures virtuelles sont présentés dans les 4 prochains paragraphes consacrés chacun à une dimension, distance ou spécification géométrique étudiée. Pour faciliter la comparaison entre les 2 méthodes, les résultats dimensionnels pour la position zéro du montage on été alignés entre le relevé réel et le calcul obtenu sur le modèle virtuel. En effet, le modèle $\mathrm{CAO}$ de la pièce diffère quelque peu du modèle réel fabriqué.

\subsection{Mesure de la déformée}

Lors de la modélisation pour les mesures virtuelles, nous avions deux options utilisables dans logiciel 3DCS: l'application d'une force localisée au niveau du point d'appui, ou la mise en coïncidence d'un point de la surface avec le point de contact mobile modélisé. La maquette réelle est réglable au niveau du positionnement du point d'appui à l'extrémité de la pièce, sans maîtrise de la force appliquée. La modélisation la plus proche de cette méthodologie expérimentale a été privilégiée. Après un premier essai en utilisant le déplacement du point de contact, nous avons observé un écart entre l'angle virtuel et l'angle mesuré. Cet écart provient du fait que le déplacement imposé sur le modèle virtuel selon l'axe Z, n'est pas le reflet exact de ce qui se passe sur la maquette réelle. En effet, lorsque le point d'appui se déplace en $\mathrm{Z}$, la pièce subit une flexion qui oblige à déplacer également l'appui en $X$ pour rester sur le même point de contact physique. Suite à cette observation, il a été décidé de 
remplacer le point de coïncidence par une force qui suit le point d'appui lors de la déformation en conservant une direction constante. Cette seconde méthode entraine le calcul préalable de la force associée au déplacement en utilisant Abaqus.

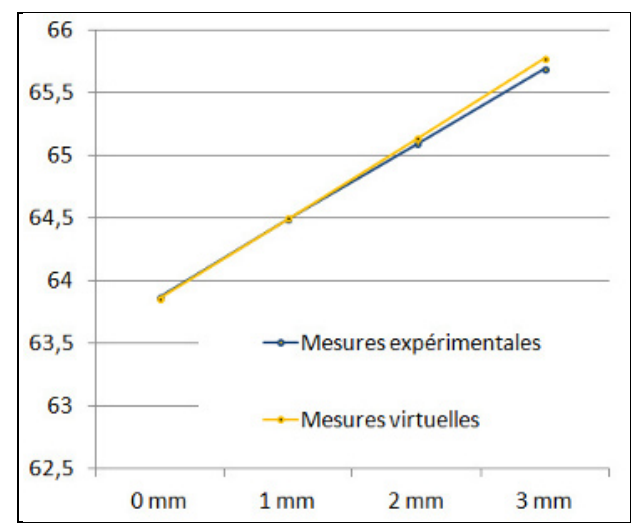

Figure 8. Mesure angulaire

La mesure de l'angle (Figure 8) entre la surface inclinée et le repère principal (plan XY de référence horizontal) a permis de valider le modèle choisi pour appliquer le déplacement local correspondant au point d'appui expérimental. Le modèle utilisé dans FEA Compliant Modeler de 3DCS ayant été validé, nous avons pu réaliser les autres mesures pour comparaison.

\subsection{Mesure de distance avec grande déformation}

La seconde mesure étudiée est l'entraxe soumis à la plus grande déformation. La mesure virtuelle de la distance est obtenue directement à l'aide de 3DCS. La concordance entre les 2 graphiques permet d'envisager l'utilisation de ce type d'outil pour prévoir les défauts concernant des tolérances de localisation.

Si l'on suppose que la pièce subit une flexion principale autour de son point de fixation et que l'amplitude de cette déformation est liée directement au déplacement de l'extrémité libre sur son point d'appui, on peut s'attendre à une diminution de la distance lorsque la déformation augmente (Figure 9).

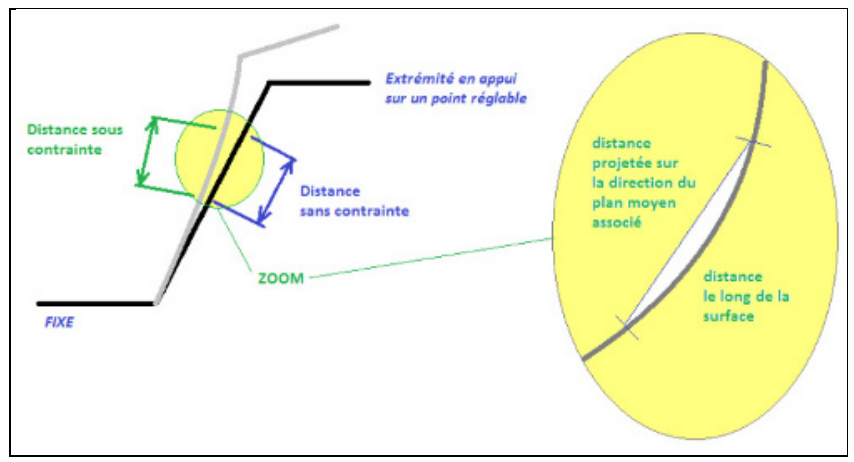

Figure 9: Variation de la distance

C'est globalement le résultat que nous obtenons (Figure 10). Le point d'inflexion indique toutefois que la distance augmente faiblement avant de suivre une pente descendante. Ce phénomène provient probablement du défaut de forme à l'état libre de la pièce qui compense au début cette variation en redressant la surface plane avant de fléchir dans la direction opposée à sa forme initiale.

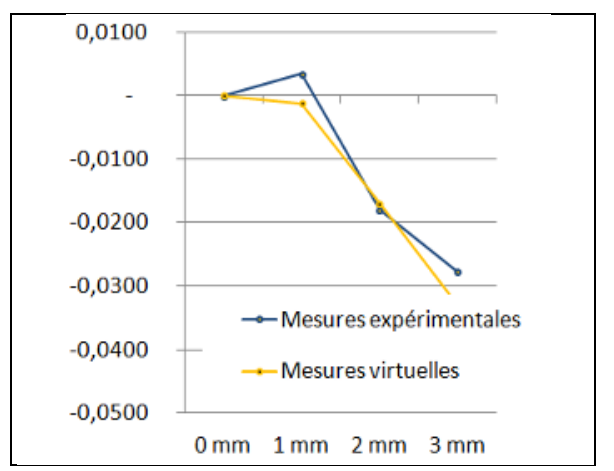

Figure 10. Mesure entraxe de $25 \mathrm{~mm}$ (incliné)

\subsection{Mesure de distance avec petite déformation}

Pour confirmer le résultat précédent, la seconde distance mesurée a été choisie dans une direction horizontale faiblement sujette à l'influence de la déformation de la pièce suite au déplacement du point d'appui. Pour un déplacement inférieur à $2 \mathrm{~mm}$ on observe une concordance des 2 graphes (Figure 11). Par contre, il apparait un écart lors de la mesure réelle, alors que la flexion de la pièce n'entraîne que très peu de déplacement dans cette direction (analyse confirmée par vérification des zones de déplacement sur le logiciel de calcul FEM Abaqus).

L'écart observé est assez faible: $0,02 \mathrm{~mm}$ L'explication de cette variation est liée à la méthode de mesure utilisée. La MMT déplace le palpeur pour mesurer les alésages en prenant comme orientation de travail le plan mesuré précédemment. Ce plan incliné est un plan moyen calculé sur l'intégralité de la surface plane. Hors, la déformation de la pièce en flexion fait que la direction utilisée n'est plus adaptée à la position locale de chaque alésage. Cette différence de plan d'orientation entraine une variation sur des points palpés et du calcul résultant.

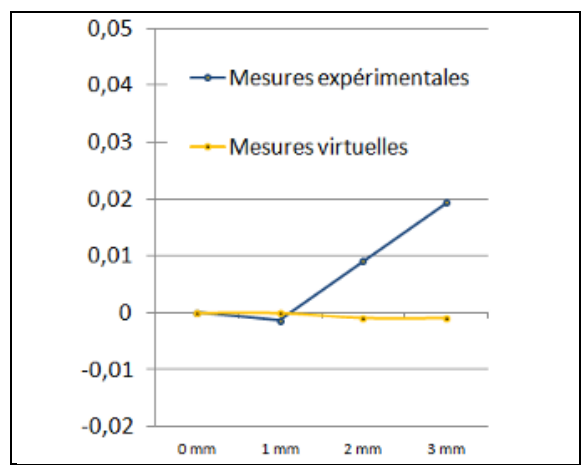

Figure 11. Mesure entraxe de $14 \mathrm{~mm}$ (horizontal)

\subsection{Mesure du défaut de planéité}

La Figure 12 présente l'évolution de la mesure du défaut de planéité de la surface plane inclinée. On peut remarquer 
que la valeur initiale pour la mesure virtuelle est à zéro. En effet, nous n'avons pas, dans un premier temps, affecté de valeur à l'annotation FTA de tolérance de forme de la surface plane. La valeur à utiliser lors d'une simulation plus complète intégrant le tolérancement géométrique de la pièce fabriquée sera égale au défaut obtenu pour la position libre, soit $0,04 \mathrm{~mm}$ de défaut de planéité pour cette pièce test.

Les deux courbes suivent la même tendance générale pour l'ensemble de l'expérimentation (déplacement du point d'appui entre 0 et $3 \mathrm{~mm}$ ). Par contre, on peut observer que la courbe représentant les mesures réelles coupe celle des mesures virtuelles. Elle présente en effet une inflexion au point $1 \mathrm{~mm}$. La cause provient du fait que la pièce réelle présente un défaut de forme initiale, mais que la déformation due au déplacement du point d'appui la redresse. L'augmentation du défaut de planéité entre le 0 et $1 \mathrm{~mm}$ est donc plus faible que par la suite. Cette écart entre le réel et le virtuel est cependant très faible comparé à l'effet de la flexion de la pièce sur le calcul du défaut de planéité : $0,04 \mathrm{~mm}$ d'écart sur un défaut maximum atteint de $0,4 \mathrm{~mm}$.

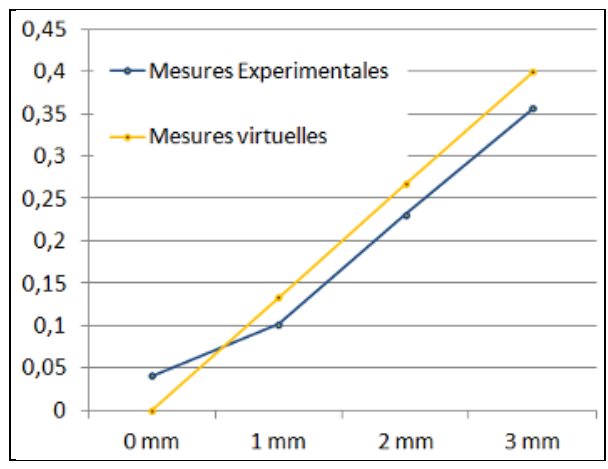

Figure 12. Mesure de planeité

\section{Conclusion}

Cette étude est une première étape dans la mise en place d'une méthode de simulation des mesures de pièces déformables. Les résultats obtenus montrent une certaine correspondance entre les mesures réelles et les mesures virtuelles. La plus grande difficulté reste de définir correctement pour chaque type de situation (mesure d'entraxe, de localisation, de défaut de forme, ...) la meilleure méthode de calcul: la plus économique en temps et difficulté de mise en place, tout en restant suffisamment proche des mesures réelles pour pouvoir obtenir des résultats fiables.

Les perspectives de cette étude sont d'évoluer vers la modélisation $\mathrm{du}$ contrôle des pièces plastiques déformables dans le domaine automobile. Une des principales difficultés concerne la prise en compte de la non-linéarité de certains matériaux et l'application de grands déplacements en dehors du domaine linéaireélastique classique. Le second point d'amélioration sera de disposer de pièces et de montages de contrôles représentatifs de la problématique afin d'appliquer les méthodes envisagées en partant du problème initial: plusieurs pièces fabriquées de dimensions différentes qui sont bridées et donc déformées sur un unique montage de contrôle.

Le dernier point d'amélioration envisagé est l'intégration des défauts de fabrication sous forme de cotation 3D (annotations FTA) en mode simultané avec les calculs de type éléments finis. Cela permettrait de passer d'une étude basée sur des mesures virtuelles, comme celle présentée ici, à une simulation prenant en compte la capabilité des procédés de fabrication pour remplacer le montage physique de contrôle par une validation numérique des mesures attendues associée à la qualité de la pièce à contrôler.

\section{Références}

1. A. Jaramillo, P. Boulanger, F. Prieto, On-line 3-D system for the inspection of deformable parts, The International Journal of Advanced Manufacturing Technology 57 (9-12) 1053-1063 (2011).

2. A. Jaramillo, P. Boulanger, F. Prieto, Fast dimensional inspection of deformable parts from partial views, Computers in Industry, In Press, 2013.

3. A. Jaramillo, P. Boulanger, F. Prieto, Deformable part inspection using a spring-mass system, ComputerAided Design 45 1128-1137 (2013).

4. E. Savio, L. De Chiffre, R. Schmitt, Metrology of freeform shaped parts, Annals of the CIRP 56(2) 810-835 (2007).

5. V. WOLFF, D-T. TRAN, S. RAYNAUD, Using virtual parts to optimize the metrology process Tehnomus 19 9-15 (2012).

6. H. Radvar-Esfahlan, S.-A. Tahan, Nonrigid geometric metrology using generalized numerical inspection fixtures, Precision Engineering 36 (1) 1-9 (2012).

7. G. Abenhaim, A. Tahan, A. Desrochers, A novel approach for the inspection of flexible parts without the use of special fixtures, Journal of Manufacturing Science and Engineering 133 (1), 011009.1-011009.11 (2011).

8. I. Gentilini, K. Shimada, Predicting and evaluating the post-assembly shape of thin-walled components via 3D laser digitization and FEA simulation of the assembly process, Computer-Aided Design 43 (3) 316-328 (2011).

9. S. Ravishankar, H. Dutt, B. Gurumoorthy, Automated inspection of aircraft parts using a modified ICP algorithm, International Journal of Advanced Manufacturing Technology 46 (1-4) 227-236 (2010).

10. A. Weckenmann, J. Weickmann, Optical inspection of formed sheet metal parts applying fringe projection systems and virtual fixation, Metrology and Measurement Systems 13 (4) 321-334 (2006).

11. H. Hua, J. Liang Z-z. Xiao, Z-z. Tang, A. Asundi, Y-x. Wanga, Four-camera videogrammetric system for 3D motion measurement of deformable object, Optics and Lasers in Engineering, 50 800-811 (2012). 